\title{
Analyzing Crash-Prone Drivers in Multiple Crashes for Better Safety Educational and Enforcement Strategies
}

\author{
Xiaoduan Sun ${ }^{1}$, Subasish Das ${ }^{1}$, Yulong $\mathrm{He}^{2}$ \\ ${ }^{1}$ Civil Engineering Department, University of Louisiana at Lafayette, Louisiana, USA \\ ${ }^{2}$ Beijing University of Technology, Beijing, China \\ Email: xsun@louisina.edu, sxd1684@louisiana.edu, ylhe@bjut.edu.cn
}

Received August 8, 2013; revised September 11; 2013; accepted October 6, 2013

Copyright (c) 2014 Xiaoduan Sun et al. This is an open access article distributed under the Creative Commons Attribution License, which permits unrestricted use, distribution, and reproduction in any medium, provided the original work is properly cited. In accordance of the Creative Commons Attribution License all Copyrights @ 2014 are reserved for SCIRP and the owner of the intellectual property Xiaoduan Sun et al. All Copyright (C) 2014 are guarded by law and by SCIRP as a guardian.

\begin{abstract}
Crash-prone drivers should be effectively targeted for various safety education and regulation programs because their over-involvement in crashes presents a big adverse effect on highway safety. By analyzing seven years of crash data from Louisiana, this paper investigates crash-prone drivers' characteristics and estimates their risk to have crashes in the seventh year based on these drivers' crash history of the past six years. The analysis results show that quite a few drivers repeatedly had crashes; seven drivers had 13 crashes in seven years; and the maximum number of crashes occurring in a single year to a single driver is eight. The probability of having crash(es) in any given year is closely related to a driver's crash history: less than $4 \%$ for drivers with no crash in the previous six years; and slightly higher than $30 \%$ for drivers with nine or more crashes in the previous six years. Based on the results, several suggestions are made on how to improve roadway safety through reducing crashes committed by drivers with much higher crash risk as identified by the analysis.
\end{abstract}

\section{KEYWORDS}

\section{Roadway Safety; Crash-Prone Driver; Crash Risk; Probability}

\section{Introduction}

Although conservatively speaking more than $50 \%$ of crashes are caused by human errors each year based on highway crash reports, engineers are always trying to make roadways more forgiving and vehicles more crashworthy, which has made considerable impact on highway safety. Under the persistent effort, highway fatal crashes in U.S. have finally reached the lowest number since 1960. Much of the effort has been spent on implementing crash countermeasures on highway facilities through enhancing safety on roadway geometric features and traffic control devices. Safety education and enforcement, the other two elements in the $4 \mathrm{E}$ approach (emergency services the fourth), also made strides in educating the general public on various safety risks and enforcing safety traffic laws.

To fulfill the hefty goal established by the AASHTO Highway Safety Strategy to cut traffic fatalities in half by 2020 and by Louisiana Strategic Highway Safety Plan for Destination Zero Deaths, it is important to have effective safety education and regulation programs while continually improving the highway infrastructure's safety. Since crash-prone drivers present a big adverse effect on highway safety, they should be effectively targeted in various safety education and enforcement programs. It is generally known that very young and very old drivers have the highest fatal crash rates, but it does not mean that these two groups committed most of crashes. People with similar personal traits could have very different crash risk. Identifying high risk drivers and studying their characteristics are critical in further reducing the number of crashes through targeted safety education and enforcement programs. Thus, a project was conducted at the University of Louisiana to study the impact of crashprone drivers on safety and to predict how a driver's past crash history could affect his/her crash occurrence(s) in the upcoming year. Most importantly, the study is to provide evidence for developing better and efficient 
safety education programs and supporting targeted traffic laws or programs on these crash over-involved drivers.

\section{Literature Review}

Investigating crash-prone drivers' characteristics, exploring the relationship between drivers' past crash/citation history and their crash risk, and predicting drivers' future crash occurrences by their previous crash history were the focus of many past studies.

The existence of crash-prone drivers was first recognized in 1920 by Greenwood and Yule. In their published paper, crash-prone drivers are defined as the drivers with higher than expected number of crashes [1]. In a 2003 study by Blasco, crash-prone drivers are described as the drivers with recurring crashes that were caused by human error, not by coincidence [2]. In 1971, Peck et al. concluded that it is quite difficult to accurately identify which driver will or will not cause crashes because of the statistical nature of crash frequencies [3]. After analyzing five years of Kentucky crash data (1993-1997), Stamatiadis found that about $2.1 \%$ of the licensed drivers who were charged with six or more points in past 2 years accounted for nearly $5.3 \%$ of all crashes [4].

Predicting a driver's crash risk based on his/her past crash and traffic offence history was the topic of many investigations. Through examining older drivers' previous conviction record and crash data, Daigneault in 2002 concluded that prior crashes are a better predictor for crash risk than prior convictions [5]. In a study published in 1991, Hauer determined that if the prediction model makes the right use of the driver's past crash records, the performance of their multivariate model for a crash would be improved [6]. A logistic regression model was developed by Chen in 1995 to identify crash prone drivers based on their records prior to their at-fault crash involvements, which discovered that a model using prior at-fault crash data can recognize up to $23 \%$ more drivers who will have one or more at-fault crash involvements in the next 2 years than a model that uses the conviction information [7]. After studying 17 logistic regression models, Gebers in 1999 concluded that his models could correctly classify crash-involved drivers up to $27.6 \%$ [8]. By deploying canonical correlation techniques in a subsequent research effort, Gebers and Peck in 2003 achieved an accuracy level up to $27.2 \%$ from their best model to identify crash-prone drivers [9]. Although no model can be considered perfect, the modeling progress can be seen in much research especially from the Californian studies [7,8]. However, some researchers have voiced their skepticism over crash-prone drivers' prediction $[3,8]$.

Although the past studies on crash-prone drivers have yielded some interesting results, the small sample size and lack of detailed data on drivers' characteristics and crashes have limited the research findings.

\section{Data Analysis}

Seven years of crash data, 2004-2010, in Louisiana are used for this analysis. There are more than one million crashes recorded in this seven year period, but about $10 \%$ of these records do not have the computer generated driver ID (meaning no driver license information collected at crash scene or hit-and-run crashes), which makes the cross-year analysis impossible. The crash records with no driver ID were removed from the analysis. The at-fault drivers, i.e., drivers responsible or mainly responsible for a crash listed as driver- 1 in the data based, were selected for the analysis. In general about 4\% of licensed drivers in Louisiana are involved in at least one crash each year. The number of drivers having crashes is summarized in Table 1 , which reveals some drivers had crashes repeatedly within one year. The annual maximum number of crashes to a single driver is eight. About $10 \%$ of crashes occurred to drivers having multiple crashes annually. By examining the seven years

Table 1. Number of drivers with crashes.

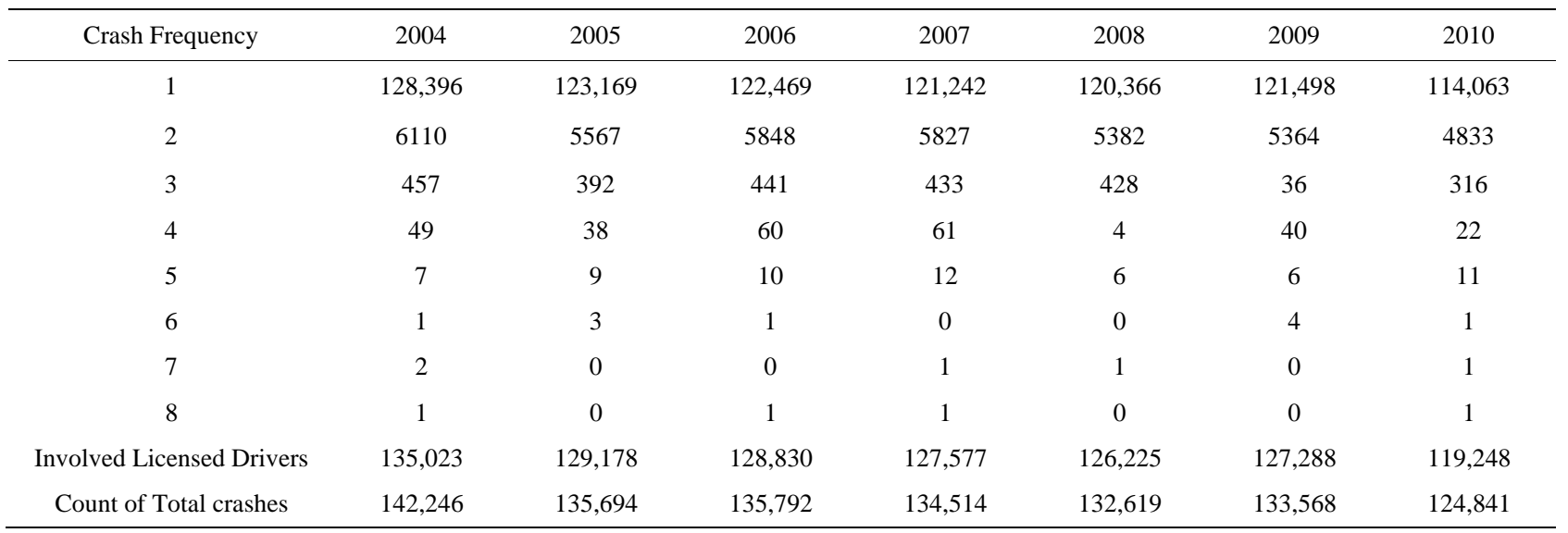


of data together, we found that the maximum number of crashes by a single driver is 13 (in 8 and more) as shown in Table 2.

As expected, the majority of crashes occurred to drivers holding a Louisiana driver license. About 66\% and 34\% of crashes are blamed on drivers with single crash and with multiple crashes in seven years, respectively. These $34 \%$ crashes were repeatedly committed by 137,812 drivers that accounts for $5 \%$ of licensed drivers in the state. As also shown in Table 3 and Figure 1, drivers with multiple crashes tend to have higher injury rate before the final fatal crash.

Table 2. Drivers' basic information.

\begin{tabular}{|c|c|c|c|c|c|c|}
\hline \multirow{2}{*}{ Crash Count } & \multirow{2}{*}{ Involved Drivers } & \multicolumn{2}{|c|}{ Gender } & \multicolumn{2}{|c|}{ Licensed by } & \multirow{2}{*}{$\%$ of Total Crashes } \\
\hline & & Male & Female & Louisiana & Others & \\
\hline 13 & 7 & $85.71 \%$ & $14.29 \%$ & $100.00 \%$ & $0.00 \%$ & $0.01 \%$ \\
\hline 12 & 5 & $100.00 \%$ & $0.00 \%$ & $100.00 \%$ & $0.00 \%$ & $0.01 \%$ \\
\hline 11 & 6 & $66.60 \%$ & $33.33 \%$ & $100.00 \%$ & $0.00 \%$ & $0.01 \%$ \\
\hline 10 & 23 & $73.91 \%$ & $26.09 \%$ & $100.00 \%$ & $0.00 \%$ & $0.02 \%$ \\
\hline 9 & 31 & $61.29 \%$ & $38.71 \%$ & $100.00 \%$ & $0.00 \%$ & $0.03 \%$ \\
\hline 8 & 95 & $77.89 \%$ & $22.11 \%$ & $100.00 \%$ & $0.00 \%$ & $0.08 \%$ \\
\hline 7 & 223 & $78.92 \%$ & $21.08 \%$ & $100.00 \%$ & $0.00 \%$ & $0.17 \%$ \\
\hline 6 & 573 & $71.73 \%$ & $28.27 \%$ & $99.83 \%$ & $0.17 \%$ & $0.37 \%$ \\
\hline 5 & 1,675 & $70.93 \%$ & $29.07 \%$ & $99.28 \%$ & $0.72 \%$ & $0.89 \%$ \\
\hline 4 & 6,139 & $68.99 \%$ & $30.85 \%$ & $80.24 \%$ & $19.76 \%$ & $2.61 \%$ \\
\hline 3 & 23,414 & $64.70 \%$ & $35.40 \%$ & $98.51 \%$ & $1.49 \%$ & $7.48 \%$ \\
\hline 2 & 105,621 & $59.36 \%$ & $40.62 \%$ & $97.09 \%$ & $2.91 \%$ & $22.49 \%$ \\
\hline 1 & 618,388 & $56.13 \%$ & $43.54 \%$ & $90.21 \%$ & $9.79 \%$ & $65.84 \%$ \\
\hline
\end{tabular}

Table 3. Distribution of crash severity.

\begin{tabular}{|c|c|c|c|c|c|c|c|c|}
\hline \multirow{2}{*}{ Crash Severity } & \multicolumn{8}{|c|}{ Crash count in Seven Years } \\
\hline & 8 and More & 7 & 6 & 5 & 4 & 3 & 2 & 1 \\
\hline Injury & 278 & 302 & 658 & 1507 & 4272 & 1879 & 34,569 & 99,534 \\
\hline PDO & 1200 & 1256 & 2768 & 6832 & 20,182 & 58,034 & 175,554 & 514,534 \\
\hline
\end{tabular}

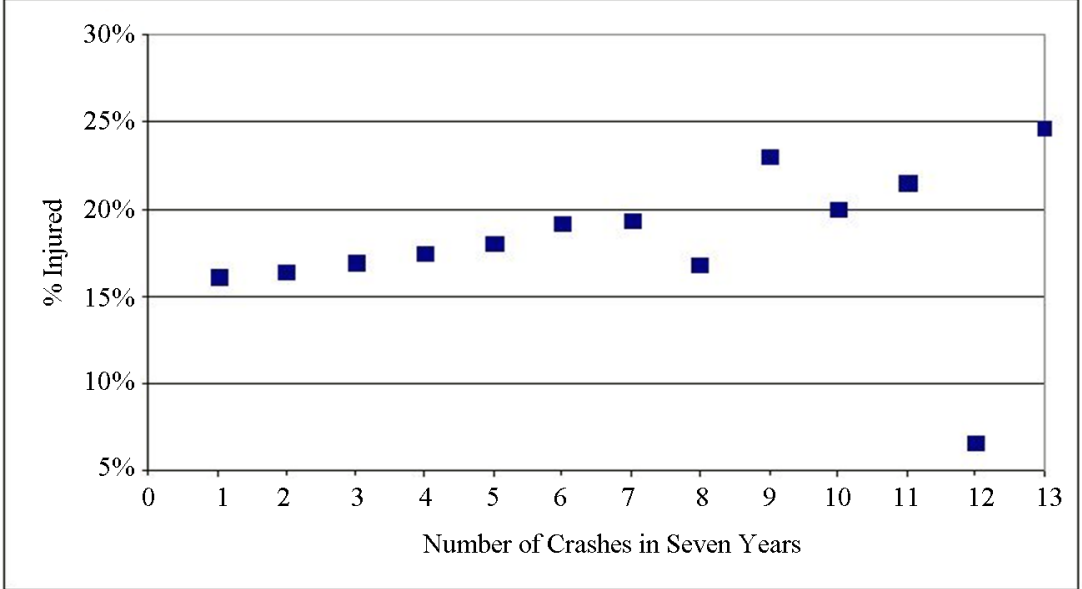

Figure 1. Distribution of injury crashes. 
The initial data analysis reveals a big variation in crash frequency among licensed drivers in Louisiana. A driver's crash risk in the coming year is closely related to the driver's safety performance. Based on a driver's crash history, we can predict the probability of having a crash or crashes in the coming year. For that purpose, two crash matrixes were developed, with each row presenting $i$ crashes in the previous six years and each column presenting $j$ crashes in the year 2010. Each cell in the first matrix presents the probability of having $j$ crashes in the seventh year and $i$ crashes in the previous six years. The summation of each column and row represents the probabilities $P(i)$ and $P(j)$. The second matrix consists of conditional probability $P(j / i)$ that is calculated by:

$$
P(j / i)=\frac{P(j \cap i)}{P(i)}
$$

With the second conditional probability matrix, the probability of having any given number of crashes, $j$, in 2010 varies by drivers' history as illustrated in Figure 2.
Thus, the probability of having crash(s) in the seventh year can be estimated as:

$$
P\left(j_{7} \geq 1 / i\right)=\sum_{j=1}^{m} \frac{P(j \cap i)}{P(i)} \text { for } i=0,1,2, \cdots, 9^{+}
$$

Based on this equation, how the probability of having crash(es) varies is illustrated in Figure 3.

It is expected that drivers with no crashes during previous six years have the lowest probability of having crash(es) in the seventh year as shown in Figure 3 and the probability of having zero crashes decreases as drivers' past crash frequency increases as shown in Figure 2. The probability of having one or two crashes in the seventh year increases as the crash frequency increases in previous years. The probability curve of having three crashes in the seventh year is different; it's increasing first and then decreases as the number of previous crashes increases.

It is interesting to know that these multiple crashes did not occur uniformly along the seven-year time period.
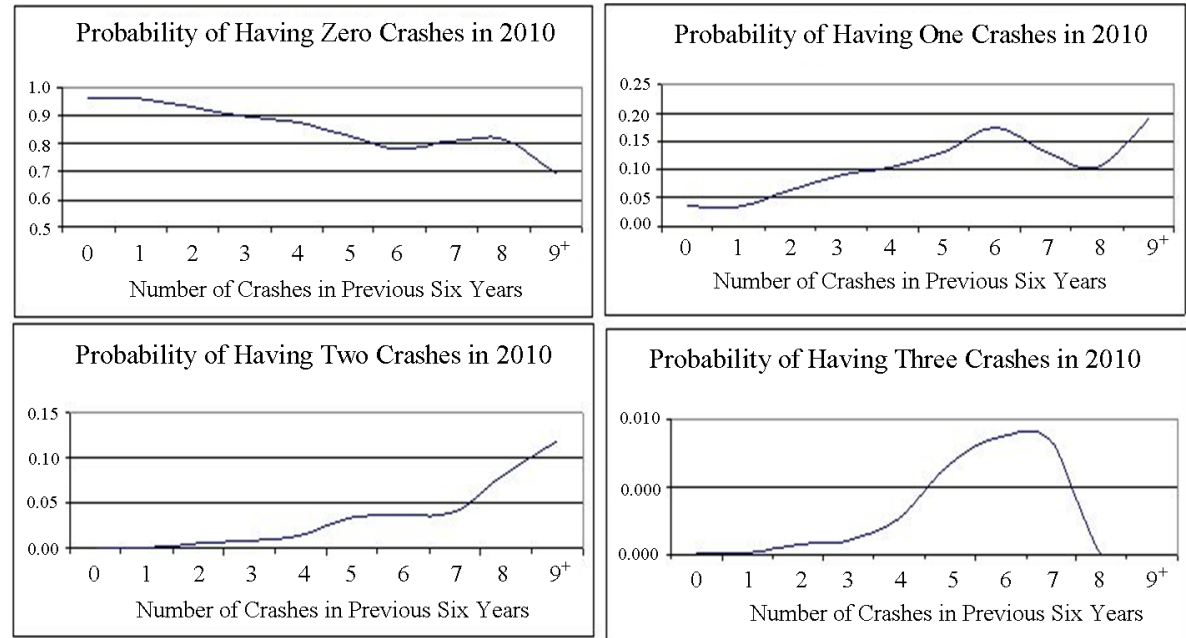

Probability of Having Three Crashes in 2010

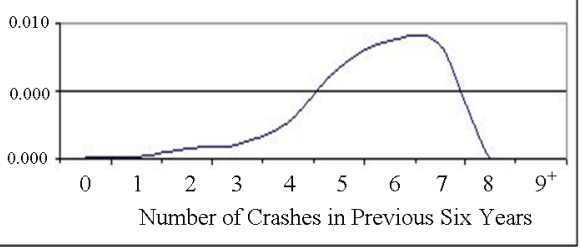

Figure 2. Probabilities of drivers having zero, one, two and three crashes.

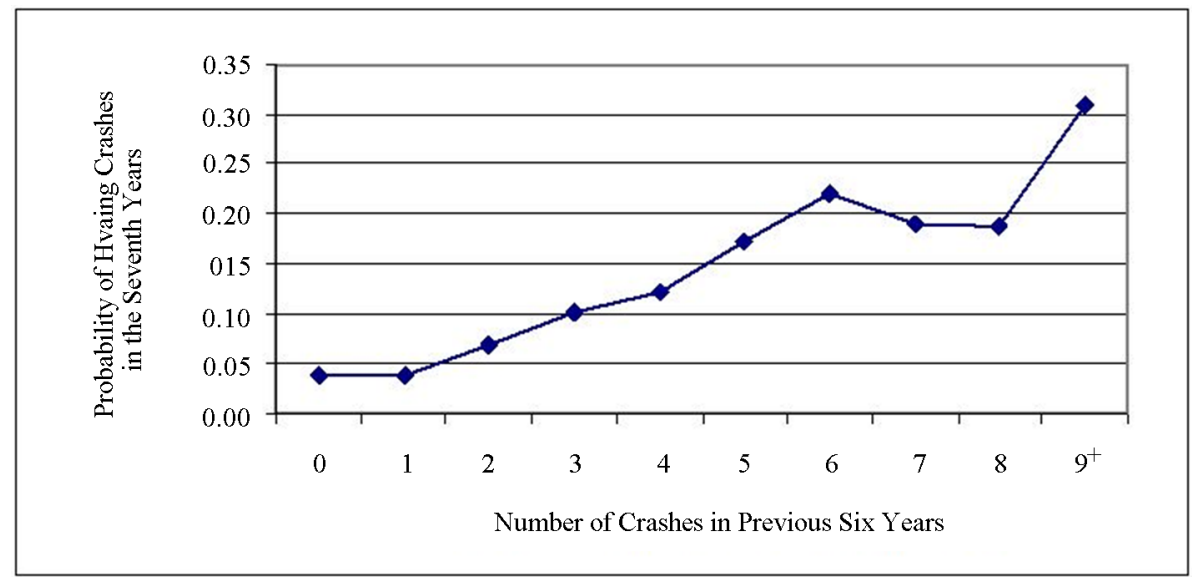

Figure 3. Conditional probability of having crash(es) in 2010. 
The gap between crashes is much smaller than the uniformly distributed gaps. As displayed in Figure 4, the estimated average gap between crashes is two years for drivers who had two crashes in the seven-year time period, which is much smaller than the uniform 3.5 years. The estimated average gap between crashes is 0.46 compared to the uniform gap of 0.54 for drivers having 13 crashes in seven years.

Crash-prone drivers apparently present a significant adverse effect on highway safety. Investigating crashprone drivers' characteristics can help to develop effective safety strategies targeting these drivers. Through the data analysis, we found the following crash characteristics are worth noting. First, the relative difference between genders by number of crashes in seven years was explored with the results shown in Table 2, which indicates the percentage of male driver increases as the number of crashes increases. About $71 \%$ of drivers with five or more crashes in seven years are male drivers while the percentage is $55 \%$ in one crash case as shown in Figure 5.

The drivers' age distribution shown in Figure 6 illustrates the differences among different age groups. One national study [10] has indicated that the very young and the very old have the highest fatal crash rate. This study reveals that the 20 to 40 age group has the highest crash involvement rate and their rate increases as the number of crashes increases.

Comparing with another age group (40 to 65 years old drivers), the over-involvement in multiple crashes by the 20 - 40 age group is more evident.

To answer the question of what are major contributing factors to these multiple crashes in seven years, we examined drivers' condition, type of violations, and type of crashes. Figure 7 displays that drug-use and distracted operation are surely responsible for multiple crashes, particularly to drivers with five and more crashes in seven years.

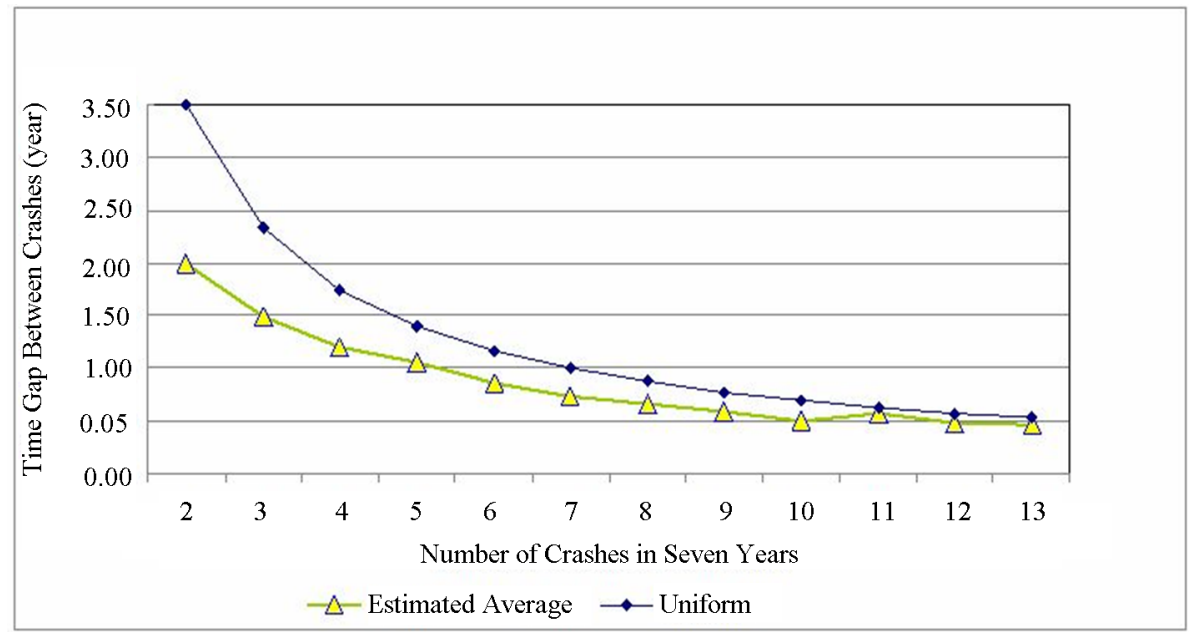

Figure 4. Time gap between crashes.

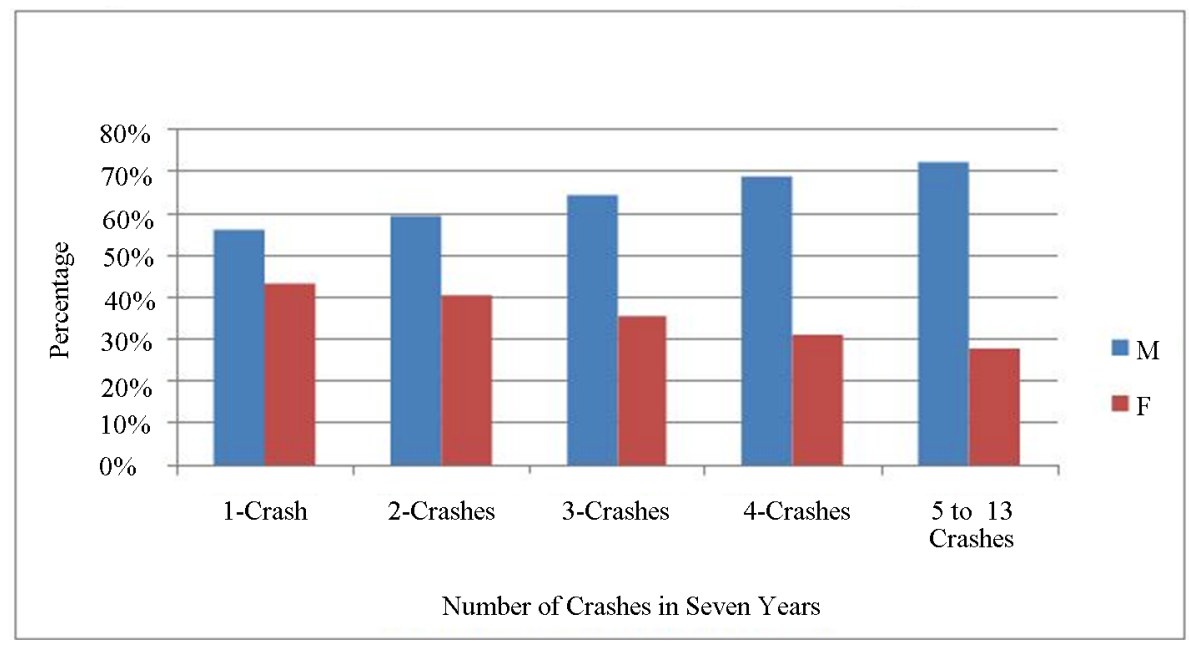

Figure 5. Distribution of drivers' gender. 
Regarding the type of violation, careless operation was cited as the main reason for the crash and it increases as the number of crashes increases as shown in Figure 8.

Rear-end collision is the most common type of crash regardless of crash frequency level. However, the percentage of rear-end collision does go up as number of crashes increase. As displayed in Figure 9, the proportion of single-vehicle crashes (non-collision with motor vehicle) also increases as number of crashes increases.

\section{Discussion and Conclusion}

The seven-year crash data analysis introduced in this paper has demonstrated that crash-prone drivers need to be carefully targeted in safety education and traffic law enforcement programs because their over-involvement in crashes presents a big adverse effect on roadway safety. The study results quantitatively confirm that $5 \%$ of drivers in Louisiana are responsible for $35 \%$ of crashes in a seven-year time period. The probability of having crash(es) in the coming year for drivers with a crash history is more then seven times higher than the probability for drivers with zero crash. These crash-prone drivers are likely to be male in the 20 - 40 age group.

Drivers with frequent crash history should be targeted for special safety programs regularly through education and regulations. For instance, a state motor vehicle registration office could work with the enforcement agencies to establish a driver license reviewing program that has authority to send warnings or to suspend a driver's license, or to request the driver to take a mandatory safety class if the driver has had multiple crashes within a short time period. These targeted safety classes should focus on distracted driving and even if these multiple crashes are not severe, our analysis shows that the next crash will most likely be severe if not fatal.

The crash-prone driver problem might be worse than the situation of drivers with incomplete information. The drivers with no valid license (suspended license) or fake

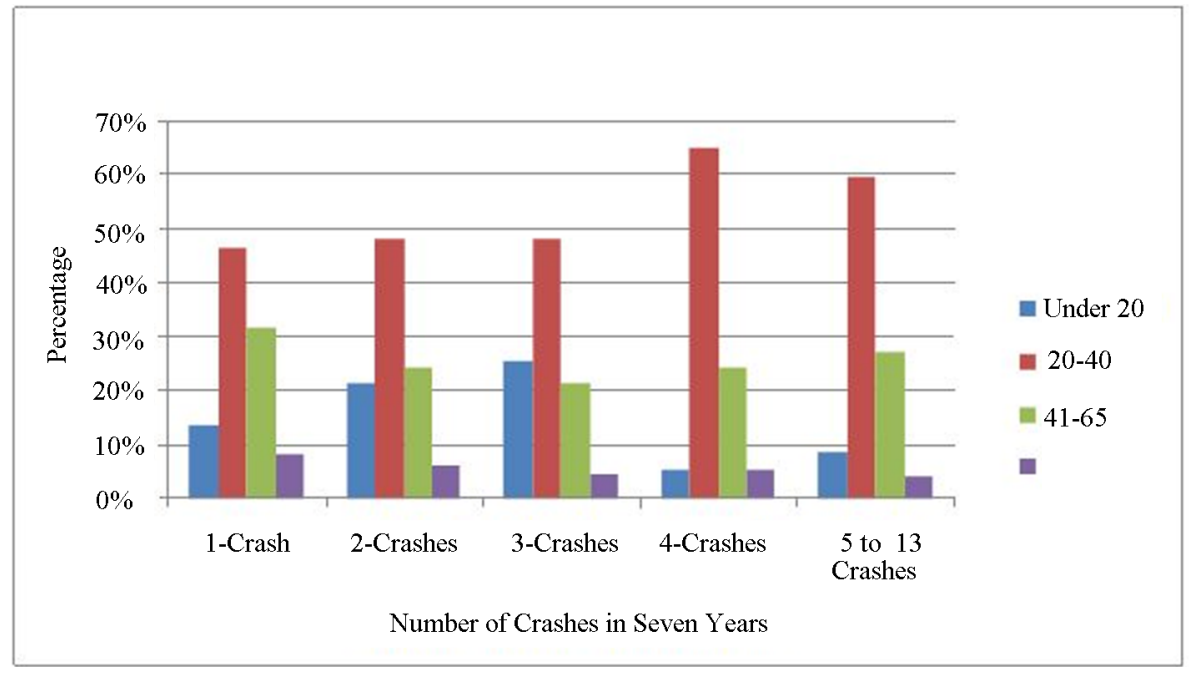

Figure 6. Distribution of drivers' age.

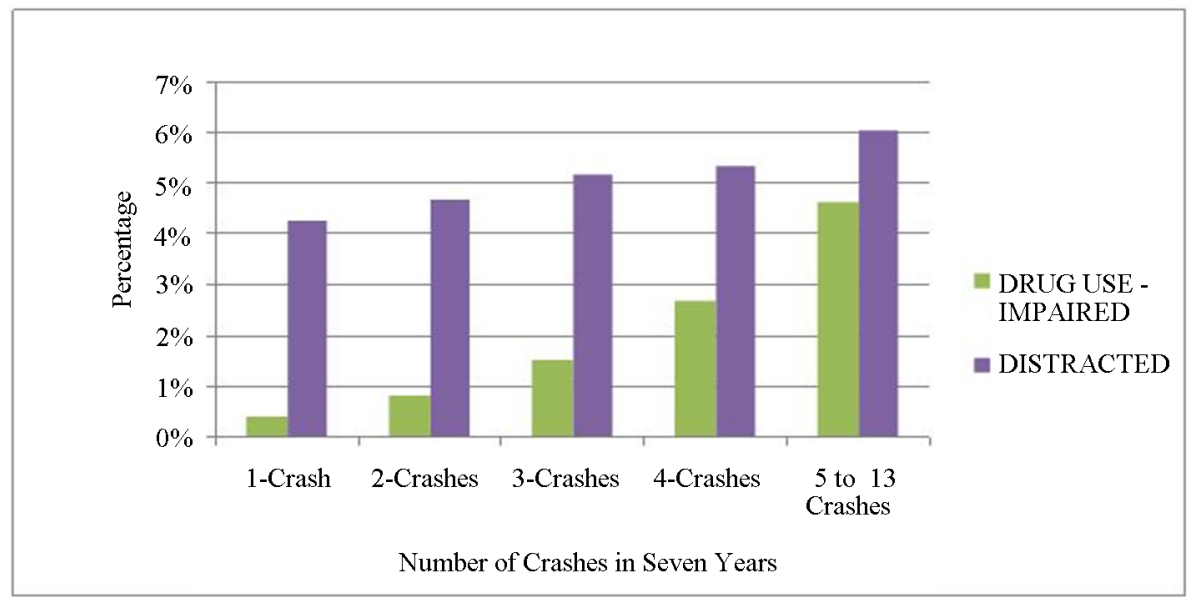

Figure 7. Drug use and distracted drivers vs. percentage of crashes. 


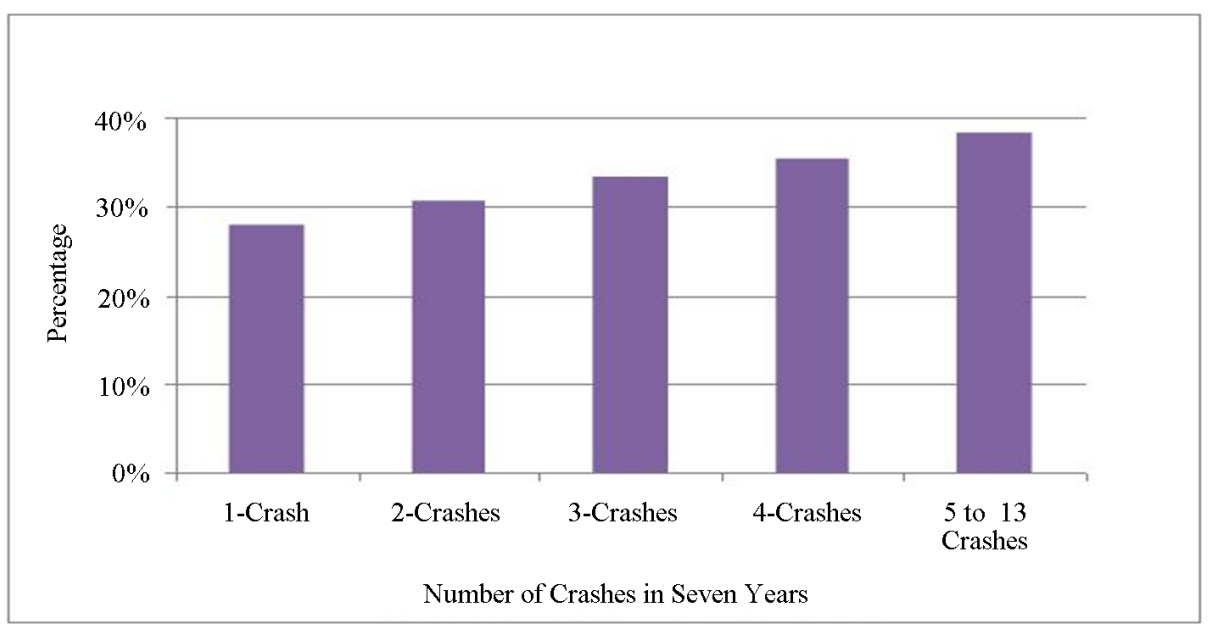

Figure 8. Careless operations vs. percentage of crashes.

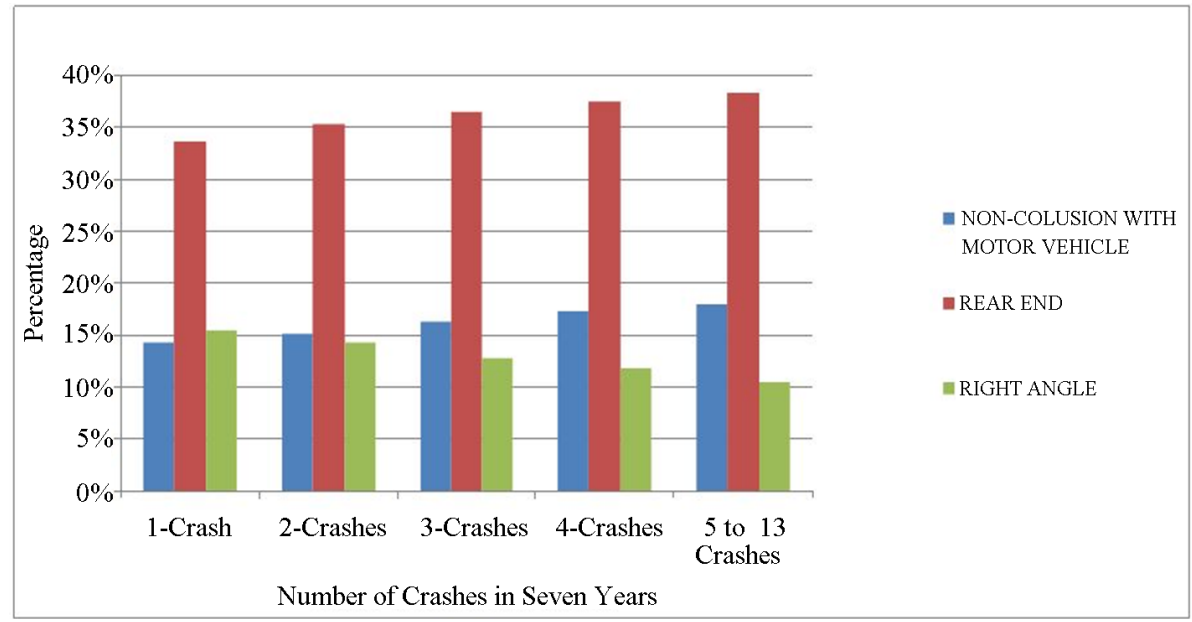

Figure 9. Type of crashes vs. percentage of crashes.

license tend to be the drivers with frequent crashes and traffic law violations.

This paper presents the initial results of a series of studies on crash-prone drivers' characteristics and individual driver's crash risk modeling. The on-going and future analysis will be extended to non-at-fault drivers involved in a crash but not responsible or less responsible for the crash. We hope that the extended analysis could shed more lights on what, how, and why these multiple crashes occur to a single driver over short time period so that more effective crash-preventive measures can be proposed to reduce recurring crashes. The analysis will also look into the crash history of drivers with a fatal crash in the last year of the analysis time period and their time gap distribution between crashes.

\section{REFERENCES}

[1] M. Greenwood and U. Yule, "An Inquiry into the Nature of Frequency Distributions Representative of Multiple
Happenings with Particular Reference to the Occurrence of Multiple Attacks of Disease or of Repeated Accidents," Journal of Royal Statistical Society, Vol. 83, No. 2, 1920, pp. 255-279. http://dx.doi.org/10.2307/2341080

[2] R. D. Blasco, J. M. Prieto and J. M. Cornejo, “Accident Probability after Accident Occurrence,” Safety Science, Vol. 4, No. 6, 2003, pp. 481-501. http://dx.doi.org/10.1016/S0925-7535(01)00080-7

[3] R. C. Peck, R. S. McBride and R. S. Coppin, "The Distribution and Prediction of Driver Accident Frequencies," Accident Analysis \& Prevention, Vol. 15, No. 5, 1971, pp. 371-393. http://dx.doi.org/10.1016/0001-4575(83)90015-5

[4] N. Stamatiadis, K. R. Agent, J. Pigman and M. Ridgeway, "Evaluation of Retesting in Kentucky's Driver License Process,” Research Report KTC-99- Kentucky Transport Cabinet, 1999.

[5] G. Daigneault, P. Joly and J. Frigon, "Previous Convictions or Accidents and the Risk of Subsequent Accidents of Older Drivers," Accident Analysis \& Prevention, Vol. 34, No. 2, 2002, pp. 257-261. http://dx.doi.org/10.1016/S0001-4575(01)00014-8 
[6] E. Hauer, B. N. Persaud, A. Smiley and D. Duncan, "Estimating the Accident Potential of an Ontario Driver," Accident Analysis \& Prevention, Vol. 23, No. 2-3, 1991, pp. 133-152.

http://dx.doi.org/10.1016/0001-4575(91)90044-6

[7] W. Chen, P. Cooper and M. Pinili, "Driver Accident Risk in Relation to the Penalty Point System in British Columbia," Accident Analysis \& Prevention, Vol. 26, No. 1, 1995, pp. 9-18.

[8] M. A. Gebers, "Strategies for Estimating Driver Accident Risk in Relation to California's Negligent-Operator Point System,” California Department of Motor Vehicles Re- search and Development Branch, Technical Monograph 183, 1999.

[9] M. A. Gebers and R. C. Peck, "Using Traffic Conviction Correlates to Identify High Accident-Risk Drivers," Accident Analysis \& Prevention, Vol. 35, No. 6, 2003, pp. 903-912.

http://dx.doi.org/10.1016/S0001-4575(02)00098-2

[10] National Highway Traffic Safety Administration (NHTSA), "An Examination of Driver Distraction as Recorded in NHTSA Databases,” Traffic Safety Facts, DOT HS 811 216, 2009. 